\title{
Protective effects of Astragalus kahiricus root extract on ethanol-induced retrograde memory impairments in mice
}

\author{
Basma M. Abdelaziz $^{\mathbb{D}}$, Maged W. Helmy, Mohamed A. Katary, Mohammad M. Abd-Alhaseeb ${ }^{* \mathbb{(}}$, Asser I. Ghoneim \\ Department of Pharmacology and Toxicology, Faculty of Pharmacy, Damanhour University, Damanhour, Egypt
}

\section{A R T I C L E I N F O}

Article Type:

Original Article

\section{Article History:}

Received: 17 March 2019

Accepted: 8 June 2019

\section{Keywords:}

Alzheimer's disease

Astragalus kahiricus

Morris water maze

Retrograde memory impairment

\begin{abstract}
A B S T R A C T
Introduction: Alzheimer's disease $(\mathrm{AD})$ is a neurodegenerative disorder that has no definite cure. Currently, there is great interest in using plant-based medicines to treat AD. In the present study, the neuroprotective effects of Astragalus kahiricus root extract were evaluated in a retrograde amnesia model.

Methods: Male albino mice were given four training sessions in the Morris water maze for seven consecutive days. Treated groups were administered A. kahiricus (25 or $50 \mathrm{mg} / \mathrm{kg}$, i.p.) before ethanol (3.5 gm/kg, i.p) injection. All animals were given a test session in the Morris water maze apparatus. Acetylcholinesterase activity and the levels of oxidative stress biomarkers were also measured.

Results: Memory impairment was observed, after ethanol administration, as increased escape latency time and path length travelled by the animals. On the other hand, A. kahiricus significantly reduced both escape latency time and path length. In addition, the extract demonstrated an inhibitory effect on acetylcholinesterase activity and total nitrite level. Moreover, A. kahiricus significantly increased the level of reduced glutathione in mice brain.

Conclusion: This study demonstrated the potential behavioural and biochemical neuroprotective properties of $A$. kahiricus root extract, which might further be considered an important candidate for the treatment of $\mathrm{AD}$.
\end{abstract}

Implication for health policy/practice/research/medical education:

The present study showed the potential behavioural and biochemical neuroprotective properties of A. kahiricus root extract, which might further be considered an important candidate for the treatment of AD.

Please cite this paper as: Abdelaziz BM, Helmy MW, Katary MA, Abd-Alhaseeb MM, Ghoneim AI. Protective effects of Astragalus kahiricus root extract on ethanol-induced retrograde memory impairments in mice. J Herbmed Pharmacol. 2019;8(4):295-301. doi: 10.15171/jhp.2019.43.

\section{Introduction}

Alzheimer's disease $(\mathrm{AD})$ is a progressive neurodegenerative brain disorder that threatens humankind. It is the most common cause of dementia in late adult life (1). It is generally characterized by the gradual and irreversible loss of memory, especially recent forms of explicit memory, confusion with place or time, increased anxiety, and numerous changes in mood and personality. The symptoms vary in severity according to the disease progression (2). The prevalence of $\mathrm{AD}$ has been estimated in many studies. Concerning the Arab population, the prevalence of dementia of the Alzheimer type (DAT) increases with age (3). The prevalence of dementia in Egypt ranges from 2.01\% to 5.07\%, increasing steeply with age, and has an estimated cost of $\$ 3109$ per person, annually (4).

Regrettably, the approved drugs for treating $\mathrm{AD}$ have serious side effects that limit their use (1). This is one of the reasons many people worldwide are turning towards alternative medicines. Recently, there has been renewed interest in plant-based medicines for the treatment of $\mathrm{AD}$ (5-7). Astragalus is the largest genus in the Fabaceae family, comprising more than 3000 species worldwide, including 35 in Egypt (8). Many Astragalus species have been used in traditional Chinese medicine as approved drugs for many years $(9,10)$. Different Astragalus species 
have been involved in many studies as neuroprotective agents, such as extracts of Astragalus mongholicus, Astragalus hamosus, and Astragalus gombiformis (11-15). In addition, the active constituent isolated from Astragalus membranaceus, astragaloside (IV) can prevent A $\beta$ 1-42 neurotoxicity in cell lines (16). Astragalus kahiricus is one type of Astragalus that has a hepatoprotective effect against ethanol-induced toxicity (8).

The hippocampus plays a crucial role in learning and memory (17). Acute ethanol exposure is a suitable method for inducing retrograde amnesia in rodents (18). It affects performance in many spatial learning and memory tasks, such as water maze test, because it produces learning and memory deficits similar to those found due to the presence of lesions in the hippocampal system in rodents $(19,20)$. Additionally, acute ethanol administration alters the function of hippocampal neurons, leading to the inhibition of basal and novel environment-induced c-Fos protein expression in the hippocampus (21). In addition, acute ethanol administration decreases glutamate release in the dorsal hippocampus and reduces hippocampal glutamate transmission, which impairs spatial memory performance (22). The present study aimed to evaluate the protective effect of $A$. kahiricus extract against memory impairment induced by acute ethanol administration.

\section{Materials and Methods}

Plant extract

The concentrated residue of $A$. kahiricus DC (Sinai, Egypt) root ethanolic extract was mixed with distilled water and successively extracted with solvents of increasing polarity, as described previously (8). The concentrated chloroformic extract was dissolved in $4 \%$ dimethyl sulfoxide (DMSO) to be tested in the present study.

\section{Chemicals}

The chemicals, i.e., acetyl thiocholine iodide, 5,5'-dithiobis(2-nitrobenzoic acid) (DTNB), reduced glutathione $(\mathrm{GSH})$, sulfanilamide and vanadium III chloride (VCL3) were purchased from Sigma-Aldrich, St. Louis, MO, USA. DMSO (99\%) and sodium nitrite (NaNO2) were purchased from Loba Chemie Pvt., Ltd., India. Ethanol (95\% W/V) was purchased from El Nasr Pharma. Co., Egypt. N-(1-Naphthyl) ethylenediamine dihydrochloride (NED) was purchased from Fluka Chemie AG. All other chemicals were of the highest grade available.

\section{Experimental animals}

Male albino mice 2-3 months old and weighing 25-30 g at the start of the study were used. Animals were housed 8 per cage, allowed access to water and food ad libitum, and maintained in suitable temperature and humidity under a 12-h light/dark cycle. All experiments were carried out in accordance with the institutional guidelines of the NIH for laboratory animal care and use, and approve from the Ethical Committee at Faculty of Pharmacy, Damanhour University with approval number 1116PO2. They were allowed to acclimate to the laboratory conditions for one week before starting the experiment. All efforts were made to minimize the number of animals used as well as their suffering. Mice were randomly distributed into 4 groups, with ten mice in each group, using a simple randomization method.

\section{Experimental design}

Animals were randomly divided into four groups, with 10 mice in each group, as follows: (1) control group, mice received normal vehicle (4\% DMSO, i.p.); (2) Eth group, mice received ethanol $20 \%(\mathrm{~W} / \mathrm{V})$ at a dose of $3.5 \mathrm{~g} / \mathrm{kg}$, i.p., on the eighth day; (3) Eth-AK 25 group, mice received A. kahiricus extract at a dose of $25 \mathrm{mg} / \mathrm{kg}$, i.p., and after 30 minutes received ethanol $20 \%(\mathrm{~W} / \mathrm{V})$ at a dose of 3.5 $\mathrm{g} / \mathrm{kg}$, i.p., on the eighth day; (4) Eth-AK 50 group, mice received Astragalus kahiricus extract at a dose of $50 \mathrm{mg} /$ $\mathrm{kg}$, i.p., and after 30 minutes received ethanol 20\% (W/V) at a dose of $3.5 \mathrm{~g} / \mathrm{kg}$, i.p., on the eighth day. Ethanol (95\% $\mathrm{W} / \mathrm{V}$ ) was dissolved in $0.9 \%$ saline at a final concentration of $20 \%(\mathrm{~W} / \mathrm{V})$.

Morris water maze apparatus and procedures

The Morris water maze offers a way of examining the neurobiology of spatial learning (23). The apparatus and the method used in this study were performed as previously described (24), with minor modifications (2527). The Morris water maze was a circular white pool (100 $\mathrm{cm}$ in diameter and $50 \mathrm{~cm}$ in height) with a featureless inner surface. The circular pool was filled with water at constant temperature $\left(23 \pm 1 \mathrm{C}^{\circ}\right) ; 500 \mathrm{~mL}$ of milk was added and mixed with the water to a height of $30 \mathrm{~cm}$. The pool was divided into four equal quadrants $(\mathrm{N}, \mathrm{E}, \mathrm{S}$ and $\mathrm{W})$. A circular white platform $(9 \mathrm{~cm}$ in diameter and $29 \mathrm{~cm}$ in height) was submerged $1 \mathrm{~cm}$ below the surface of water and centred in one of the four quadrants of the pool. The position of the platform remained constant throughout the experiment. The first day of training was dedicated to free swimming training for 60 seconds in the absence of the platform to test the mice for any motor disabilities, in which case they would be excluded. For the following seven consecutive days, the mice were given four trials each day with an inter-trial interval of 20 minutes, with each trial beginning from a different starting position $(\mathrm{N}, \mathrm{E}, \mathrm{S}$ and $\mathrm{W})$, to reach a steady escape latency. During each trial session, the mouse was placed in the water facing the wall of the tank to minimize bias. During each trial, the escape latencies of mice in reaching platform were measured using a stopwatch and were recorded by the same experimenter. This parameter was averaged for each session of trials and for each mouse. Each trial session lasted 120 seconds, and once the mouse located 
the platform, it was permitted to remain on it for 20 seconds. If the mouse did not locate the platform within 120 seconds, it was guided to the platform and allowed to stay on the platform for 20 seconds. The mouse was then removed from the pool, dried and placed in its cage. Mice that had an escape latency of more than 15 seconds on the seventh day were excluded.

On the eighth day, after the training course was complete, mice were given $A$. kahiricus extract (either 25 $\mathrm{mg} / \mathrm{kg}$ or $50 \mathrm{mg} / \mathrm{kg}$, i.p.) according to their group, and after 30 minutes, all mice except those in the control group were given $20 \%$ (W/V) ethanol (3.5 g/kg, i.p.). After 30 minutes, the animals were given a test session to reach the platform, and the mean escape latency was measured. If the mouse could not find the platform within 120 seconds, it was removed from the water and returned it to its home cage, and a latency of 121 seconds was recorded (28). The swimming path length $(\mathrm{cm})$ of each mouse was measured (29). Finally, the platform was removed (probe test), and the mice were allowed 90 seconds of free swimming; the time spent in the target quadrant (quadrant containing the platform) was measured (30).

\section{Biochemical tests}

Determination of acetylcholinesterase (AChE) activity After the Morris water maze test sessions, mice were sacrificed by rapid decapitation. The skull was opened, and the whole brain was removed. Half of the brain (which was divided symmetrically) was used to assess AChE activity according to the method described previously (31), with minor modifications, as previously reported (32). The percent inhibition of AChE was calculated relative to the control.

\section{Determination of GSH level}

GSH was assayed spectrophotometrically according to the method described previously (33), with slight modifications, as previously reported (8).

\section{Determination of the total nitrite level}

The nitrite level was assayed by a colorimetric method using Griess reagent (N-naphthyl ethylenediamine dihydrochloride and sulfanilamide), according to previous studies $(34,35)$. Brain homogenate samples were deproteinized by adding acetonitrile, followed by centrifugation at $4000 \mathrm{rpm}$ for 30 minutes; the supernatant was then recovered for nitrite determination. Then, $800 \mu \mathrm{L}$ of VCL3 was added to $800 \mu \mathrm{L}$ of the supernatant, followed by the addition of Griess reagent. The sample was mixed and incubated at $37^{\circ} \mathrm{C}$ for 30 minutes, and the absorbance of the pink-coloured product at $540 \mathrm{~nm}$ was measured using a PerkinElmer Lambda $2 S$ spectrophotometer. The concentration of nitrite (nmol nitrite/mg tissue) in the supernatant was determined using a sodium nitrite standard curve.
Statistical analysis

The results are expressed as the mean \pm S.E. The Kolmogorov-Smirnov test was used to check the normality of the data set. One-way ANOVA was used for multiple comparisons, and post hoc analysis between groups was performed with the Tukey test. The criterion for statistical significance was $P<0.01$. Correlations between variables were evaluated using Pearson's coefficient. These tests were performed using SPSS version $15 \mathrm{ev}$.

\section{Results}

Morris water maze behavioural test

The data depicted in Figure 1A show improvement in all animals throughout all days of the experiment; animals that showed any motor disabilities or difficulties in the learning of were excluded. The Pearson correlation coefficients were calculated to demonstrate the degree of association between the escape latency time (second) and days of training. A negative correlation was demonstrated between the escape latency time (second) and days of training, with a correlation coefficient $(r)$ of -0.827 at $P<$ 0.01 . The latency time was significantly (13.6-fold) higher in the Eth group than in the control group $(P<0.01)$. The animals in the Eth+AK ( 25 or $50 \mathrm{mg} / \mathrm{kg}$ ) group had significantly shorter latency times (by 3.4- and 9.6-fold, respectively) than animals in the Eth group $(P<0.01)$. The travelled path length was significantly increased (by 4.8 fold) in the Eth group compared with the control group. Animals in the Eth+AK group (25 or $50 \mathrm{mg} / \mathrm{kg}$ ) had significantly shorter path lengths (by 1.7 - and 4.2-fold, respectively) than those in the Eth group $(P<0.01$, Figure $1 \mathrm{~B}$ and $1 \mathrm{C})$. As shown in Figure 1D, the animals in the Eth group spent significantly less time in the target quadrant than did animals in the control group during the probe trial $(P<0.01)$. However, the animals in the Eth+AK $(25$ or $50 \mathrm{mg} / \mathrm{kg}$ ) groups demonstrated a significant preference for the target quadrant $(P<0.01)$.

Biochemical tests

\section{Determination of AChE activity}

The acute intraperitoneal administration of ethanol had no effect on the brain AChE level compared with the control group. In contrast, the intraperitoneal injection of Eth+AK extract ( 25 or $50 \mathrm{mg} / \mathrm{kg}$ ) resulted in a significant decline in brain AChE activity compared with that in both the control and Eth groups $(P<0.01$, Figure 2$)$.

\section{Determination of the GSH level}

As shown in Figure 3, the acute administration of ethanol had no effect on the brain GSH level $(231.02 \pm 8.05 \mu \mathrm{g} / \mathrm{g}$ tissue) compared to the control $(274.3 \pm 13.8 \mu \mathrm{g} / \mathrm{g}$ tissue; $P$ $<0.01)$. However, pretreatment with Astragalus kahiricus extract at 25 or $50 \mathrm{mg} / \mathrm{kg}$ caused a significant increase in the GSH level $(386.1 \pm 13.5$ and $514.44 \pm 17.19 \mu \mathrm{g} / \mathrm{g}$ tissue, respectively, $P<0.01)$ compared to both the control and ethanol treatment. 

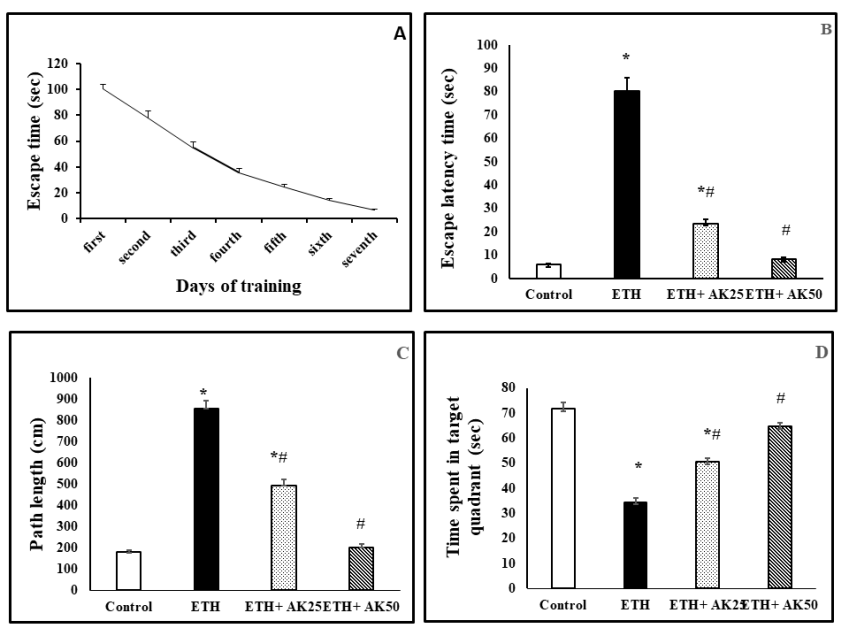

Figure 1. Effect of Astragalus kahiricus extract ( 25 or $50 \mathrm{mg} / \mathrm{kg}$, i.p) in ethanol induced memory impairment model by using Morris water maze test. (A) Correlation between the escape latency time (second) and the days of training sessions (B) Escape latency time (second), (C) Path length $(\mathrm{cm})$ travelled and (D) Time spent in target quadrant (second). The results are expressed as the mean \pm S.E ( $n=10)$. One-way ANOVA was used for multiple comparisons, and post hoc analysis between groups was performed with the Tukey test. *significantly different from control group at $(P<0.01)$. \#significantly different from ethanol group at $P<0.01$.

\section{Determination of the total nitrite level}

The acute intraperitoneal administration of ethanol caused a significant increase in the brain nitrite level (2509.36 $\pm 77.13 \mathrm{nmol} / \mathrm{g}$ tissue) compared to the control group $(1371.88 \pm 45.19 \mathrm{nmol} / \mathrm{g}$ tissue; $P<0.01)$. Pretreatment with $A$. kahiricus extract resulted in a significant reduction in the nitrite level, with nitrite levels of $1826.83 \pm 40.79$ and $1436.08 \pm 64.83 \mathrm{nmol} / \mathrm{g}$ tissue in the Eth+AK 25 and Eth + AK 50 groups, respectively (both $P<0.01$ compared with the Eth group; Figure 4).

\section{Discussion}

The present study investigated the effect of A. kahiricus extract in preventing retrograde memory impairment induced by the acute intraperitoneal administration of ethanol. The core findings of this study are as follows: (i) pretreatment with different doses of $A$. kahiricus extract counteracted the impairment in the Morris water maze test performance caused by acute ethanol exposure; (ii) pretreatment with A. kahiricus extract resulted in a significant decrease in the enzyme activity of AChE in the rodent brain despite AChE activity not being affected by acute ethanol administration; and (iii) pretreatment with A. kahiricus extract resulted in a significant reduction in the levels of oxidative stress biomarkers, as indicated by an increase in the GSH level and a decrease in the nitrite level, which had been greatly increased by acute ethanol administration.

It has been reported that the acute administration of ethanol results in learning and memory impairments in different spatial memory tasks, such as the Morris water maze, similar to those produced following lesions to the hippocampal system in rodents $(19,20,28)$. The present study demonstrated that the intraperitoneal injection of $20 \% \mathrm{~W} / \mathrm{V}$ ethanol $(3.5 \mathrm{gm} / \mathrm{kg}) 30$ minutes before the Morris water maze test session induced significant spatial memory impairment, which was reversed by pretreatment with $A$. kahiricus extract.

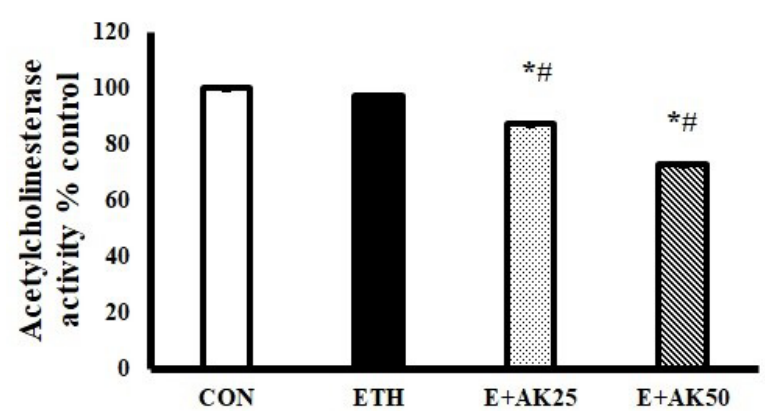

Figure 2. Effect of Astragalus kahiricus extract (25 or $50 \mathrm{mg} / \mathrm{kg}$, i.p) on Acetylcholinesterase activity in ethanol induced memory impairment model. *Significantly different from control group at $(P<0.01)$. \#Significantly different from ethanol group at $(P<0.01)$.

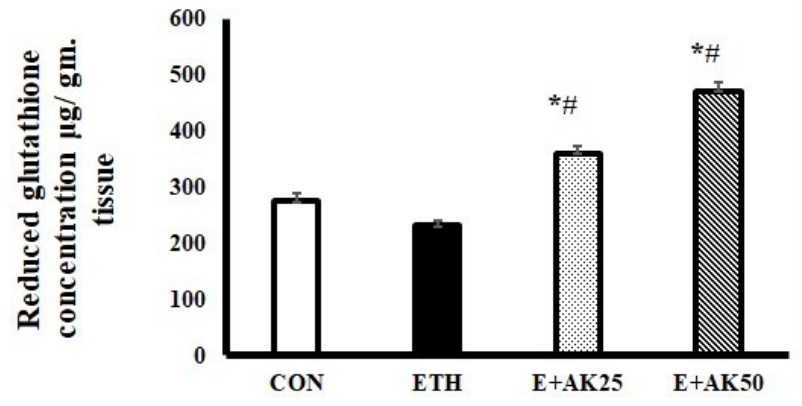

Figure 3. Effect of Astragalus kahiricus extract ( 25 or $50 \mathrm{mg} / \mathrm{kg}$, i.p) on reduced glutathione level in ethanol induced memory impairment model. *Significantly different from control group at $(P<0.01)$. " Significantly different from ethanol group at $(P<0.01)$. 


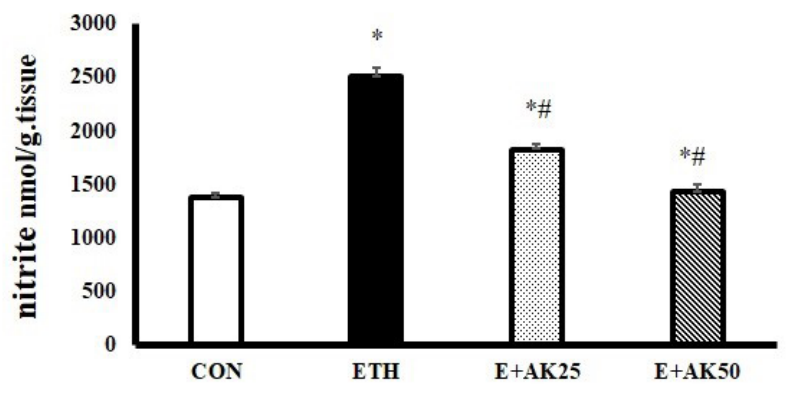

Figure 4. Effect of Astragalus kahiricus extract (25 or $50 \mathrm{mg} / \mathrm{kg}$, i.p) on total nitrite levels in ethanol induced memory impairment model. *Significantly different from control group at $(P<0.01)$. "Significantly different from ethanol group at $(P<0.01)$.

Both the escape latency time and path length travelled by mice to reach the platform position were significantly increased in ethanol-treated mice compared to control mice. However, in the probe test, the time spent in the target quadrant was significantly decreased in ethanoltreated mice compared to control mice, and these results are in accordance with the findings of other studies (20, 28).

Pretreatment with A. kahiricus extract (25 or $50 \mathrm{mg} /$ $\mathrm{kg}) 30$ minutes before the ethanol treatment counteracted this memory impairment by reversing the effect of ethanol on spatial memory in the Morris water maze test. This pretreatment markedly decreased both the escape latency $(\mathrm{sec})$ time and the path length $(\mathrm{cm})$ travelled by mice to reach the platform position. However, in the probe trial, the administration of $A$. kahiricus extract (25 or $50 \mathrm{mg} / \mathrm{kg}) 30$ minutes before the administration of ethanol significantly increased the time spent in the target quadrant. These results are supported by the findings of previous studies on another species of Astragalus $(12,36)$.

The oldest theory in AD pathophysiology is the cholinergic hypothesis, which posits that deficits in the neurotransmitter acetylcholine contribute to the symptoms of the disease (37). The present results demonstrated, for the first time, that acute ethanol administration at a dose of $3.5 \mathrm{~g} / \mathrm{kg}$ caused no significant change in AChE activity relative to the control due to the effects of dose and time. In contrast, chronic ethanol administration to rodents reduces the level of the neurotransmitter acetylcholine in the rodent brains by either reducing the synthesis and release of acetylcholine (38) or increasing AChE activity in the brain tissue $(39,40)$. The administration of $A$. kahiricus extract ( 25 or $50 \mathrm{mg} / \mathrm{kg}$ ) 30 minutes before the administration of ethanol markedly decreased the activity of AChE in a dose-dependent manner compared to that in both the control and Eth groups, revealing that $A$. kahiricus extracts have anticholinesterase activity. Notably, A. gombiformis extracts inhibit AChE activity (15).

Oxidative stress is considered to be the main factor in the process of ageing and to play a role in the pathophysiology of $\mathrm{AD}(41,42)$. GSH is critical for protecting the brain from oxidative stress by acting as a free radical scavenger; thus, an increase in the GSH level is beneficial in AD. In this study, a non-significant difference was found in the brain GSH level between mice in the Eth and control groups. However, a higher dose of acute alcohol intake can reduce the GSH level by $84 \%$ (43). In addition, the chronic administration of ethanol to rodents results in a significant decrease in the glutathione level in the brain tissue $(39,40,44)$. The administration of different doses of A. kahiricus extract 30 minutes before the administration of ethanol markedly increased the brain GSH level compared to those in both the control and Eth groups. These findings are in accordance with the results of a previous study on A. kahiricus in 2013 revealing that extracts of $A$. kahiricus increased the level of GSH in the liver tissue after the induction of apoptosis by ethanol (8). Additionally, previous studies have demonstrated that isoflavonoids from A. mongholicus have antioxidant activities, including via glutathione peroxidase (GSH-Px) (13).

Nitric oxide has significant physiological functions, and nitric oxide pathways have been implicated in a number of neurological disorders, including AD (45). In the present study, acute ethanol administration significantly increased the brain nitrite level compared with the control. Previous studies have shown that the chronic administration of ethanol markedly increased the nitrite levels in the brain tissue of rodents $(39,40)$. However, pretreatment with different doses of $A$. kahiricus extract affected this increase in a dose-dependent manner.

The neuroprotective effects of A. kahiricus extract may be attributed to cycloartane triterpenes (astrasieversianin II, astramembrannin II, astrasieversianin XIV and cycloastragenol) and cycloartane type saponins (kahiricosides II-V) $(8,46,47)$, which have been isolated from this plant in previous studies.

\section{Conclusion}

In summary, the multitude of effects of A. kahiricus extract justifies the search for potential neuroprotective mechanisms involved in improving the memory impairment and cognitive disabilities observed in AD. A. kahiricus extract exhibits considerable activity against cholinesterase and oxidative stress, both of which play a critical role in the pathophysiology of AD. A. kahiricus extract may be a promising agent for the treatment of $\mathrm{AD}$ and could even delay the progression of this notorious disorder.

\section{Acknowledgments}

Researchers at the Department of Pharmacognosy, Faculty of Pharmacy, University of Alexandria, Egypt, are acknowledged for kindly supplying the plant extract as previously investigated in collaboration (8). 


\section{Ethical approval}

All experiments were carried out in accordance with the institutional guidelines of the NIH for laboratory animal care and use, and approved in the Ethical Committee at Faculty of Pharmacy, Damanhour University, with approval number 1116PO2.

\section{Competing interests}

The authors have no conflicts of interest to disclose.

\section{Funding/Support}

None.

\section{References}

1. Kumar A, Singh A, Ekavali. A review on Alzheimer's disease pathophysiology and its management: an update. Pharmacol Rep. 2015;67(2):195-203. doi: 10.1016/j. pharep.2014.09.004.

2. 2016 Alzheimer's disease facts and figures. Alzheimers Dement. 2016;12(4):459-509.

3. Bowirrat A, Treves TA, Friedland RP, Korczyn AD. Prevalence of Alzheimer's type dementia in an elderly Arab population. Eur J Neurol. 2001;8(2):119-23.

4. Elshahidi MH, Elhadidi MA, Sharaqi AA, Mostafa A, Elzhery MA. Prevalence of dementia in Egypt: a systematic review. Neuropsychiatr Dis Treat. 2017;13:715-20. doi: $10.2147 /$ ndt.s127605.

5. Perry NS, Bollen C, Perry EK, Ballard C. Salvia for dementia therapy: review of pharmacological activity and pilot tolerability clinical trial. Pharmacol Biochem Behav. 2003;75(3):651-9.

6. Anekonda TS. Resveratrol--a boon for treating Alzheimer's disease? Brain Res Rev. 2006;52(2):316-26. doi: 10.1016/j. brainresrev.2006.04.004.

7. Hamaguchi T, Ono K, Yamada M. REVIEW: Curcumin and Alzheimer's disease. CNS Neurosci Ther. 2010;16(5):28597. doi: 10.1111/j.1755-5949.2010.00147.x.

8. Allam RM, Selim DA, Ghoneim AI, Radwan MM, Nofal SM, Khalifa AE, et al. Hepatoprotective effects of Astragalus kahiricus root extract against ethanol-induced liver apoptosis in rats. Chin J Nat Med. 2013;11(4):354-61. doi: 10.1016/s1875-5364(13)60052-7.

9. Sinclair S. Chinese herbs: a clinical review of Astragalus, Ligusticum, and Schizandrae. Altern Med Rev. 1998;3(5):338-44.

10. Pistelli L, Bertoli A, Lepori E, Morelli I, Panizzi L. Antimicrobial and antifungal activity of crude extracts and isolated saponins from Astragalus verrucosus. Fitoterapia. 2002;73(4):336-9.

11. Yu LJ, Wuliji A, Bao SY, Gong GH. Anti-aging and enhanced physique activities research of Astragalus mongolicus water extract in mice. Clin Exp Pharmacol. 2015;5(3):175. doi: 10.4172/2161-1459.1000175.

12. Tohda C, Tamura T, Matsuyama S, Komatsu K. Promotion of axonal maturation and prevention of memory loss in mice by extracts of Astragalus mongholicus. Br J Pharmacol. 2006;149(5):532-41. doi: 10.1038/sj.bjp.0706865.

13. Yu D, Duan Y, Bao Y, Wei C, An L. Isoflavonoids from Astragalus mongholicus protect PC12 cells from toxicity induced by L-glutamate. J Ethnopharmacol. 2005;98(12):89-94. doi: 10.1016/j.jep.2004.12.027.

14. Bahaeddin Z, Yans A, Khodagholi F, Sahranavard S. Neuroprotection and anxiety like behavior reduction of Allium hirtifolium and Astragalus hamosus in the $\mathrm{A} \beta$ injected rat. Res J Pharmacogn. 2016;3(4):39-49. [Persian].

15. Teyeb H, Mabrouk H, Neffati M, Douki W, Najjar MF. Anticholinesterase activity of Astragalus gombiformis extracts. Journal of Biologically Active Products from Nature. 2011;1(5-6):344-8. doi: 10.1080/22311866.2011.10719103.

16. Sun Q, Jia N, Wang W, Jin H, Xu J, Hu H. Protective effects of astragaloside IV against amyloid beta1-42 neurotoxicity by inhibiting the mitochondrial permeability transition pore opening. PLoS One. 2014;9(6):e98866. doi: 10.1371/ journal.pone.0098866.

17. Duvernoy HM. The human hippocampus: functional anatomy, vascularization and serial sections with MRI. Springer Science \& Business Media; 2005.

18. Spinetta MJ, Woodlee MT, Feinberg LM, Stroud C, Schallert K, Cormack LK, et al. Alcohol-induced retrograde memory impairment in rats: prevention by caffeine. Psychopharmacology (Berl). 2008;201(3):361-71. doi: 10.1007/s00213-008-1294-5.

19. Matthews DB, Ilgen M, White AM, Best PJ. Acute ethanol administration impairs spatial performance while facilitating nonspatial performance in rats. Neurobiol Learn Mem. 1999;72(3):169-79. doi: 10.1006/nlme.1998.3900.

20. Berry RB, Matthews DB. Acute ethanol administration selectively impairs spatial memory in $\mathrm{C} 57 \mathrm{BL} / 6 \mathrm{~J}$ mice. Alcohol. 2004;32(1):9-18. doi: 10.1016/j. alcohol.2003.09.005.

21. Ryabinin AE, Criado JR, Henriksen SJ, Bloom FE, Wilson MC. Differential sensitivity of c-Fos expression in hippocampus and other brain regions to moderate and low doses of alcohol. Mol Psychiatry. 1997;2(1):32-43.

22. Shimizu K, Matsubara K, Uezono T, Kimura K, Shiono H. Reduced dorsal hippocampal glutamate release significantly correlates with the spatial memory deficits produced by benzodiazepines and ethanol. Neuroscience. 1998;83(3):701-6.

23. Morris R. Spatial localization does not require the presence of local cues. Learn Motiv. 1981;12(2):239-60. doi: 10.1016/0023-9690(81)90020-5.

24. Morris R. Developments of a water-maze procedure for studying spatial learning in the rat. J Neurosci Methods. 1984;11(1):47-60.

25. Jeong EJ, Lee KY, Kim SH, Sung SH, Kim YC. Cognitiveenhancing and antioxidant activities of iridoid glycosides from Scrophularia buergeriana in scopolamine-treated mice. Eur J Pharmacol. 2008;588(1):78-84. doi: 10.1016/j. ejphar.2008.04.015.

26. Kang SY, Lee KY, Park MJ, Kim YC, Markelonis GJ, Oh $\mathrm{TH}$, et al. Decursin from Angelica gigas mitigates amnesia induced by scopolamine in mice. Neurobiol Learn Mem. 2003;79(1):11-8.

27. Quillfeldt JA. Behavioral methods to study learning and memory in rats. In: Andersen ML, Tufik S, eds. Rodent model as tools in ethical biomedical research. Cham: Springer International Publishing; 2016:271-311.

28. Matthews DB, Morrow AL, Tokunaga S, McDaniel JR. 
Acute ethanol administration and acute allopregnanolone administration impair spatial memory in the Morris water task. Alcohol Clin Exp Res. 2002;26(11):1747-51. doi: 10.1097/01.alc.0000037219.79257.17.

29. Hosseini M, Mohammadpour T, Karami R, Rajaei Z, Sadeghnia HR, Soukhtanloo M. Effects of the hydroalcoholic extract of Nigella sativa on scopolamine-induced spatial memory impairment in rats and its possible mechanism. Chin J Integr Med. 2015;21(6):438-44. doi: 10.1007/s11655-014-1742-5.

30. Moosavi M, Khales GY, Abbasi L, Zarifkar A, Rastegar K. Agmatine protects against scopolamine-induced water maze performance impairment and hippocampal ERK and Akt inactivation. Neuropharmacology. 2012;62(5-6):201823. doi: 10.1016/j.neuropharm.2011.12.031.

31. Ellman GL, Courtney KD, Andres V Jr, Feather-Stone RM. A new and rapid colorimetric determination of acetylcholinesterase activity. Biochem Pharmacol. 1961;7:88-95. doi: 10.1016/0006-2952(61)90145-9.

32. Ragab HM, Ashour HMA, Galal A, Ghoneim AI, Haidar HR. Synthesis and biological evaluation of some tacrine analogs: study of the effect of the chloro substituent on the acetylcholinesterase inhibitory activity. Monatshefte für Chemie. 2016;147(3):539-52. doi: 10.1007/s00706-0151641-2.

33. Ellman GL. Tissue sulfhydryl groups. Arch Biochem Biophys. 1959;82(1):70-7.doi:10.1016/0003-9861(59)90090-6.

34. Guevara I, Iwanejko J, Dembinska-Kiec A, Pankiewicz J, Wanat A, Anna P, et al. Determination of nitrite/nitrate in human biological material by the simple Griess reaction. Clin Chim Acta. 1998;274(2):177-88. doi: 10.1016/s00098981(98)00060-6.

35. Miranda KM, Espey MG, Wink DA. A rapid, simple spectrophotometric method for simultaneous detection of nitrate and nitrite. Nitric Oxide. 2001;5(1):62-71. doi: 10.1006/niox.2000.0319.

36. Li WZ, Li WP, Zhang W, Yin YY, Sun XX, Zhou SS, et al. Protective effect of extract of Astragalus on learning and memory impairments and neurons' apoptosis induced by glucocorticoids in 12-month-old male mice. Anat Rec (Hoboken). 2011;294(6):1003-14. doi: 10.1002/ar.21386.

37. Francis PT, Palmer AM, Snape M, Wilcock GK. The cholinergic hypothesis of Alzheimer's disease: a review of progress. J Neurol Neurosurg Psychiatry. 1999;66(2):13747. doi: 10.1136/jnnp.66.2.137.

38. Arendt T, Allen Y, Marchbanks RM, Schugens MM, Sinden J, Lantos PL, et al. Cholinergic system and memory in the rat: effects of chronic ethanol, embryonic basal forebrain brain transplants and excitotoxic lesions of cholinergic basal forebrain projection system. Neuroscience. 1989;33(3):43562.

39. Tiwari V, Chopra K. Resveratrol prevents alcoholinduced cognitive deficits and brain damage by blocking inflammatory signaling and cell death cascade in neonatal rat brain. J Neurochem. 2011;117(4):678-90. doi: 10.1111/j.1471-4159.2011.07236.x.

40. Silvers JM, Tokunaga S, Mittleman G, O’Buckley T, Morrow AL, Matthews DB. Chronic intermittent ethanol exposure during adolescence reduces the effect of ethanol challenge on hippocampal allopregnanolone levels and Morris water maze task performance. Alcohol. 2006;39(3):151-8. doi: 10.1016/j.alcohol.2006.09.001.

41. Sastre J, Pallardo FV, Vina J. Glutathione, oxidative stress and aging. Age. 1996;19(4):129-39. doi: 10.1007/ bf02434082.

42. Markesbery WR. Oxidative stress hypothesis in Alzheimer's disease. Free Radic Biol Med. 1997;23(1):134-47.

43. Guerri C, Grisolia S. Changes in glutathione in acute and chronic alcohol intoxication. Pharmacol Biochem Behav. 1980;13 Suppl 1:53-61.

44. Raghavendra V, Kulkarni SK. Possible antioxidant mechanism in melatonin reversal of aging and chronic ethanol-induced amnesia in plus-maze and passive avoidance memory tasks. Free Radic Biol Med. 2001;30(6):595-602.

45. Fernandez AP, Pozo-Rodrigalvarez A, Serrano J, MartinezMurillo R. Nitric oxide: target for therapeutic strategies in Alzheimer's disease. Curr Pharm Des. 2010;16(25):2837-50.

46. Radwan MM, El-Sebakhy NA, Asaad AM, Toaima SM, Kingston DG. Kahiricosides II-V, cycloartane glycosides from an Egyptian collection of Astragalus kahiricus. Phytochemistry 2004;65:2909-13.

47. Verotta L, Guerrini M, El-Sebakhy NA, Assad AM, Toaima SM, et al. Cycloartane and oleanane saponins from Egyptian Astragalus spp. as modulators of lymphocyte proliferation. Planta Med. 2002;68:986-94. 